\title{
On the Gauss Map of Surfaces of Revolution with Nonlightlike Axis in Minkowski 3-Space
}

\author{
Minghao Jin ${ }^{1,2}$ and Donghe Pei ${ }^{1}$ \\ ${ }^{1}$ School of Mathematics and Statistics, Northeast Normal University, Changchun 130024, China \\ ${ }^{2}$ Department of Mathematics, Heilongjiang Institute of Technology, Harbin 150050, China
}

Correspondence should be addressed to Donghe Pei; peidh340@nenu.edu.cn

Received 16 December 2013; Accepted 27 January 2014; Published 9 March 2014

Academic Editor: Jaume Giné

Copyright (c) 2014 M. Jin and D. Pei. This is an open access article distributed under the Creative Commons Attribution License, which permits unrestricted use, distribution, and reproduction in any medium, provided the original work is properly cited.

\begin{abstract}
We study surfaces of revolution with a nonlightlike axis in 3-dimensional Minkowski space and classify such surfaces in terms of the Gauss map $G$ that satisfies the condition $\Delta^{h} G=\Lambda G$, with $\Lambda$ being a $3 \times 3$ real matrix. Furthermore, this paper completes the classification problem of surfaces of revolution in Minkowski 3-space given by Jin et al. (2013).
\end{abstract}

\section{Introduction}

The notion of finite type immersions introduced by Chen in [1] has been widely used in studying submanifolds of Euclidean and pseudo-Euclidean spaces. Also, such a notion can be extended to smooth maps on submanifolds. Among them the Gauss map is a very useful and extensively used to deal with submanifolds [2].

Let $M$ be a connected surface in Euclidean 3-space $\mathbb{R}^{3}$, and let $G: M \rightarrow \mathbb{S}^{2} \subset \mathbb{R}^{3}$ be its Gauss map. It is well known [3] that $M$ has constant mean curvature if and only if $\Delta G=\|\Lambda\|^{2} G$, with $\Delta$ being the Laplace operator on $M$ corresponding to the induced metric on $M$ from $\mathbb{R}^{3}$. As a special case, one can consider Euclidean surfaces whose Gauss map is an eigenfuction of the Laplacian; that is,

$$
\Delta G=\lambda G, \quad \lambda \in \mathbb{R} .
$$

On the other hand, Chen and Piccinni [2] proved that the only compact surface in a Euclidean 3-space satisfying (1) is a sphere. Jang [4] studied that an orientable, connected surface in a Euclidean 3-space satisfying (1) is a sphere or a circular cylinder. On the generalization of (1), Dillen et al. [5] studied surfaces of revolution in a Euclidean 3-space $\mathbb{R}^{3}$ such that their Gauss map $G$ satisfies the condition

$$
\Delta G=\Lambda G, \quad \Lambda \in \operatorname{Mat}(3, \mathbb{R})
$$

and proved that such surfaces are part of the planes, the spheres, and the circular cylinders.

As a Lorentz version of Dillen et al.s result, the author proves the following [6].

Theorem 1. The only spacelike or timelike surfaces of revolution in $\mathbb{R}_{1}^{3}$ whose Gauss map $G: M \rightarrow M^{2}(\varepsilon)$ satisfies (2) are locally the following spaces:

(1) $\mathbb{R}_{1}^{2}, S_{1}^{1} \times R^{1}$ and $\mathbb{R}_{1}^{1} \times S^{1}$ if $\varepsilon=1$,

(2) $\mathbb{R}^{2}, H^{2}$ and $H^{1} \times \mathbb{R}^{1}$ if $\varepsilon=-1$.

Recently, we studied [7] such surface and its Gauss map satisfy the following condition in Minkowski space:

$$
\Delta^{h} G=\Lambda G, \quad \Lambda \in \operatorname{Mat}(3, \mathbb{R}),
$$

where $\Delta^{h}$ is the Laplace operator with respect to the second fundamental form $h$ of the surface. This operator is formally defined by

$$
\Delta^{h}=-\frac{1}{\sqrt{|\mathscr{H}|}} \sum_{i, j=1}^{2} \frac{\partial}{\partial x^{i}}\left(\sqrt{|\mathscr{H}|} h^{i j} \frac{\partial}{\partial x^{j}}\right)
$$

for the components $h_{i j}(i, j=1,2)$ of the second fundamental form $h$ on $M$, and we denote by $\left(h^{i j}\right)$ (resp., $\mathscr{H}$ ) the inverse matrix (resp., the determinant) of the matrix $\left(h_{i j}\right)$. 
The main purpose of this note is to complete classification of surfaces of revolution in $\mathbb{R}_{1}^{3}$ whose Gauss map satisfies the condition $\Delta^{h} G=\Lambda G$. Actually, we will show the de Sitter pseudosphere, the hyperbolic pseudosphere, and five kinds of catenoid satisfying the above condition.

\section{Preliminaries}

Let $\mathbb{R}_{1}^{3}$ be a 3-dimensional Minkowski space with the scalar product and Lorentz cross product defined as

$$
\begin{gathered}
\langle\mathbf{x}, \mathbf{y}\rangle=-x_{0} y_{0}+x_{1} y_{1}+x_{2} y_{2}, \\
\mathbf{x} \times \mathbf{y}=\left(x_{2} y_{1}-x_{1} y_{2}, x_{2} y_{0}-x_{0} y_{2}, x_{0} y_{1}-x_{1} y_{0}\right)
\end{gathered}
$$

for any vectors $\mathbf{x}=\left(x_{0}, x_{1}, x_{2}\right)$ and $\mathbf{y}=\left(y_{0}, y_{1}, y_{2}\right)$ in $\mathbb{R}_{1}^{3}$.

A vector $\mathbf{x}$ of $\mathbb{R}_{1}^{3}$ is said to be spacelike if $\langle\mathbf{x}, \mathbf{x}\rangle>0$ or $\mathbf{x}=\mathbf{0}$, timelike if $\langle\mathbf{x}, \mathbf{x}\rangle\langle 0$, and lightlike or null if $\langle\mathbf{x}, \mathbf{x}\rangle=0$ and $\mathbf{x} \neq \mathbf{0}$. A timelike or lightlike vector in $\mathbb{R}_{1}^{3}$ is said to be causal. Let $\gamma: I \rightarrow \mathbb{R}_{1}^{3}$ be a smooth curve in $\mathbb{R}_{1}^{3}$, where $I$ is an interval in $\mathbb{R}$. We call $\gamma$ spacelike, timelike, or lightlike curve if the tangent vector $\gamma^{\prime}$ at any point is spacelike, timelike, or lightlike, respectively.

Let $I$ be an open interval and $\gamma: I \rightarrow \Pi$ a plane curve lying in a plane $\Pi$ of $\mathbb{R}_{1}^{3}$ and $l$ a straight line in $\Pi$ which does not intersect with the curve $\gamma$. A surface of revolution $M$ with axis $l$ in $\mathbb{R}_{1}^{3}$ is defined to be invariant under the group of motions in $\mathbb{R}_{1}^{3}$, which fixes each point of the line $l$ [8]. From this we obtain four kinds of surfaces of revolution in $\mathbb{R}_{1}^{3}$. If the axis $l$ is timelike (resp., spacelike), then there is a Lorentz transformation by which the axis $l$ is transformed to the $x_{0}$-axis (resp., $x_{1}$-axis or $x_{2}$-axis). Hence, without loss of generality, we may consider as the axis of revolution with the $x_{0}$-axis or $x_{2}$-axis if $l$ is not null. If the axis is null, then we may assume that this axis is the line spanned by vector $(1,1,0)$ on the plane $O x_{0} x_{1}$.

We now introduce three different types of surfaces of revolution in $\mathbb{R}_{1}^{3}$.

Type 1. The surface of revolution with timelike axis.

Without loss of generality, we choose $x_{0} \mathrm{O}$ as the axis. Meanwhile suppose that $\gamma$ has a parameter as follows:

$$
\gamma(u)=(g(u), f(u), 0),
$$

where $f(u)$ and $g(u)$ are smooth functions and $f(u)>0$. Then the surface of revolution $M$ with $x_{0}$-axis may be given by

$$
x(u, v)=(g(u), f(u) \cos v, f(u) \sin v) .
$$

Type 2. The surface of revolution with spacelike axis.

Without loss of generality, we choose $x_{2} \mathrm{O}$ as the axis, and suppose that curve $\gamma$ has a parameter as follows:

$$
\gamma(u)=(0, f(u), g(u)) \quad \text { or } \quad \gamma(u)=(f(u), 0, g(u)),
$$

where $f(u)$ and $g(u)$ are smooth functions and $f(u)>0$. Then the surface of revolution $M$ with $x_{2}$-axis may be given by

$$
x(u, v)=(f(u) \sinh v, f(u) \cosh v, g(u))
$$

or

$$
x(u, v)=(f(u) \cosh v, f(u) \sinh v, g(u)) .
$$

Type 3. The surface of revolution with lightlike axis.

Without loss of generality, we choose a line spanned by the vector $(1,1,0)$ as axis, and suppose that curve $\gamma$ has a parameter as follows:

$$
\gamma(u)=(f(u), g(u), 0),
$$

where $f(u)$ is smooth positive function and $g(u)$ is smooth function such that $h(u)=f(u)-g(u) \neq 0$. Then the surface of revolution $M$ with the line spanned by vector $(1,1,0)$ as axis may be given by

$$
x(u, v)=\left(f(u)+\frac{v^{2}}{2} h(u), g(u)+\frac{v^{2}}{2} h(u), h(u) v\right) .
$$

Here we only consider type 1 and type 2, as for type 3 we have already discussed in [7].

Now, let us consider the Gauss map $G$ on a surface $M$ in $\mathbb{R}_{1}^{3}$. The map $G: M \rightarrow Q^{2}(\varepsilon) \subset \mathbb{R}_{1}^{3}$ which sends each point of $M$ to the unit normal vector to $M$ at that point is called the Gauss map of surface $M$. Here $\varepsilon(= \pm 1)$ denotes the sign of the vector field $G$ and $Q^{2}(\varepsilon)$ is a 2-dimensional space form as follows:

$$
Q^{2}(\varepsilon)= \begin{cases}S_{1}^{2}(1) & \text { in } \mathbb{R}_{1}^{3} \text { if } \varepsilon=1 \\ H^{2}(-1) & \text { in } \mathbb{R}_{1}^{3} \text { if } \varepsilon=-1\end{cases}
$$

A surface $M \subset \mathbb{R}_{1}^{3}$ is called minimal if and only if mean curvature $H=0$. Now we consider some examples of minimal surfaces which will be mentioned in theorems.

Example 2 (the catenoid of the 1st kind is shown in Figure 1). A surface of catenoid of the 1st kind is parameterized by

$$
x(u, v)=(u, \sinh u \cos v, \sinh u \sin v)
$$

for $\sinh u>0$. Then the components of the first and the second fundamental forms are given by

$$
\begin{array}{ccc}
g_{11}=\sinh ^{2} u, & g_{12}=g_{21}=0, & g_{22}=\sinh ^{2} u, \\
h_{11}=-1, & h_{12}=h_{21}=0, & h_{22}=1 .
\end{array}
$$

So the mean curvature $H$ on the surface is

$$
H=\frac{(-1) \sinh ^{2} u+1 \sinh ^{2} u}{2 \sinh ^{2} u \sinh ^{2} u}=0 .
$$

Therefore, the surface of catenoid of the 1st kind is minimal. 


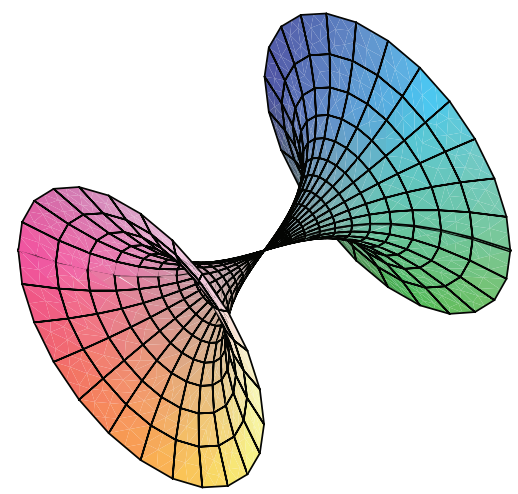

FIGURE 1: The catenoid of the 1st kind.

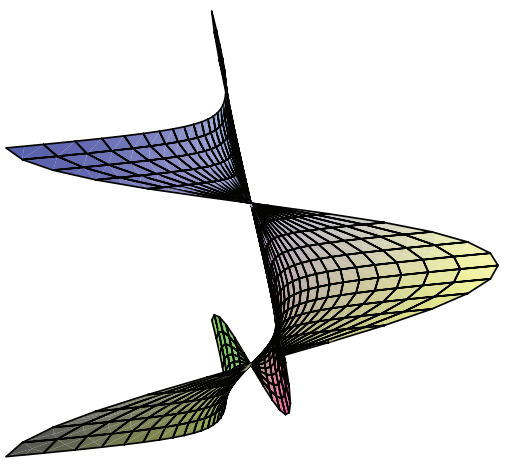

Figure 2: The catenoid of the 2nd kind.

Example 3 (the catenoid of the 2nd kind is shown in Figure 2). A surface of catenoid of the 2nd kind is parameterized by

$$
x(u, v)=(\cos u \cosh v, \cos u \sinh v, u)
$$

for $\cos u>0$. Then the components of the first and the second fundamental forms are given by

$$
\begin{array}{ccc}
g_{11}=\cos ^{2} u, & g_{12}=g_{21}=0, & g_{22}=\cos ^{2} u, \\
h_{11}=-1, & h_{12}=h_{21}=0, & h_{22}=1 .
\end{array}
$$

So the mean curvature $H$ on the surface is

$$
H=\frac{(-1) \cos ^{2} u+1 \cos ^{2} u}{2 \cos ^{2} u \cos ^{2} u}=0 .
$$

Therefore, the surface of catenoid of the 2nd kind is minimal.

Example 4 (the catenoid of the 3rd kind is shown in Figure 3). A surface of catenoid of the 3rd kind is parameterized by

$$
x(u, v)=(u, \cos u \cos v, \cos u \sin v)
$$

for $\cos u>0$. Then the components of the first and the second fundamental forms are given by

$$
\begin{aligned}
& g_{11}=-\cos ^{2} u, \quad g_{12}=g_{21}=0, \quad g_{22}=\cos ^{2} u, \\
& h_{11}=\cos ^{2} u, \quad h_{12}=h_{21}=0, \quad h_{22}=\cos ^{2} u \text {. }
\end{aligned}
$$

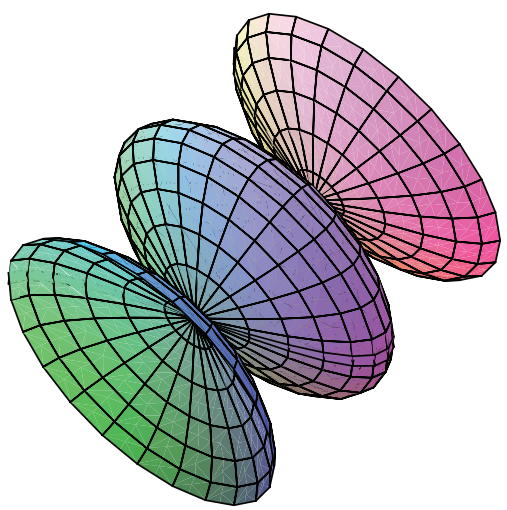

Figure 3: The catenoid of the 3rd kind.

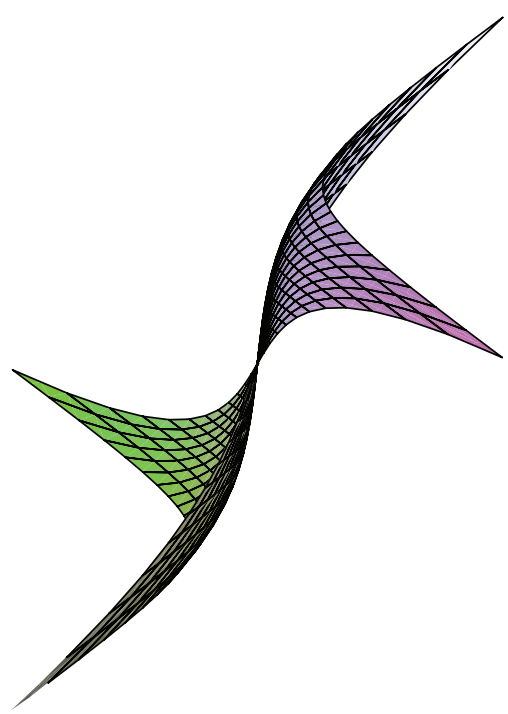

Figure 4: The catenoid of the 4th kind.

So the mean curvature $H$ on the surface is

$$
H=\frac{\cos ^{2} u \cos ^{2} u+\cos ^{2} u\left(-\cos ^{2} u\right)}{2\left(-\cos ^{2} u\right) \cos ^{2} u}=0 .
$$

Therefore, the surface of catenoid of the 3rd kind is minimal.

Example 5 (the catenoid of the 4th kind is shown in Figure 4). A surface of catenoid of the 4 th kind is parameterized by

$$
x(u, v)=(\sinh u \cosh v, \sinh u \sinh v, u)
$$

for $\sinh u>0$. Then the components of the first and the second fundamental forms are given by

$$
\begin{array}{ccc}
g_{11}=-\sinh ^{2} u, & g_{12}=g_{21}=0, & g_{22}=\sinh ^{2} u, \\
h_{11}=-1, & h_{12}=h_{21}=0, & h_{22}=-1 .
\end{array}
$$

So the mean curvature $H$ on the surface is

$$
H=\frac{(-1) \sinh ^{2} u+(-1)\left(-\sinh ^{2} u\right)}{2\left(-\sinh ^{2} u\right) \sinh ^{2} u}=0 .
$$

Therefore, the surface of catenoid of the 4th kind is minimal. 


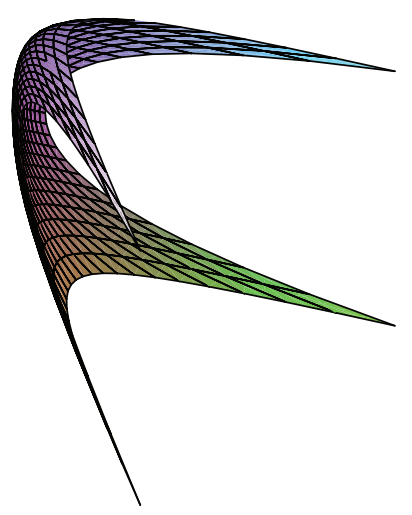

FIGURE 5: The catenoid of the 5th kind.

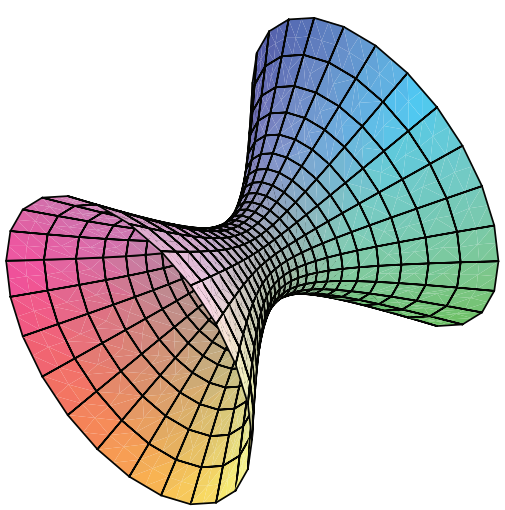

FIgURE 6: The de Sitter pseudosphere.

Example 6 (the catenoid of the 5th kind is shown in Figure 5). A surface of catenoid of the 5 th kind is parameterized by

$$
x(u, v)=(\cosh u \sinh v, \cosh u \cosh v, u)
$$

for $\cosh u>0$. Then the components of the first and the second fundamental forms are given by

$$
\begin{array}{ccc}
g_{11}=\cosh ^{2} u, & g_{12}=g_{21}=0, & g_{22}=-\cosh ^{2} u, \\
h_{11}=1, & h_{12}=h_{21}=0, & h_{22}=1 .
\end{array}
$$

So the mean curvature $H$ on the surface is

$$
H=\frac{1\left(-\cosh ^{2} u\right)+1 \cosh ^{2} u}{2 \cosh ^{2} u\left(-\cosh ^{2} u\right)}=0 .
$$

Therefore, the surface of catenoid of the 5 th kind is minimal.

Example 7 (the de Sitter pseudosphere is shown in Figure 6). The de Sitter pseudosphere centered at $(0,0,0)$ with radius 1 is parameterized by

$$
x(u, v)=(\sinh u, \cosh u \cos v, \cosh u \sin v) .
$$

Then its Gauss map $G$ and Laplacian are given by

$$
\begin{gathered}
G=(-\sinh u,-\cosh u \cos v,-\cosh u \sin v), \\
\Delta^{h}=\frac{\partial^{2}}{\partial u^{2}}-\frac{1}{\cosh ^{2} u} \frac{\partial^{2}}{\partial v^{2}}+\frac{\sinh u}{\cosh u} \frac{\partial}{\partial u} .
\end{gathered}
$$

By a straight computation, we get

$$
\Delta^{h} G=(-2 \sinh u,-2 \cosh u \cos v,-2 \cosh u \sin v) .
$$

So we have

$$
\Delta^{h} G=\left(\begin{array}{ccc}
2 & 0 & 0 \\
0 & 2 & 0 \\
0 & 0 & 2
\end{array}\right) G
$$

that is, the de Sitter pseudosphere satisfies condition (3).

\section{The Surface of Revolution with Timelike Axis}

In this section, we will classify the surfaces of revolution with timelike axis in $\mathbb{R}_{1}^{3}$ that satisfy condition (3).

Theorem 8. The only surfaces of revolution with timelike axis in $\mathbb{R}_{1}^{3}$ whose Gauss map $G$ satisfies

$$
\Delta^{h} G=\Lambda G, \quad \Lambda \in \operatorname{Mat}(3, \mathbb{R})
$$

are locally the catenoid of the 1st kind, the catenoid of the 3rd kind, the de Sitter pseudosphere, or the hyperbolic pseudosphere.

Proof. Let $M$ be a surface of revolution with timelike axis as (7). We may assume that the profile curve $\gamma$ is of unit speed; thus

$$
\left\langle\gamma^{\prime}, \gamma^{\prime}\right\rangle=-g^{\prime 2}(u)+f^{\prime 2}(u)=\varepsilon( \pm 1) .
$$

We will give detailed proof just for the case $\varepsilon=1$. Then $M$ is a spacelike surface and we may put

$$
f^{\prime}(u)=\cosh t, \quad g^{\prime}(u)=\sinh t
$$

for the smooth function $t=t(u)$. Using the natural frame $\left\{x_{u}, x_{v}\right\}$ of $M$ defined by

$$
\begin{aligned}
x_{u} & =\left(g^{\prime}, f^{\prime} \cos v, f^{\prime} \sin v\right), \\
x_{v} & =(0,-f \sin v, f \cos v), \\
x_{u u} & =\left(g^{\prime \prime}, f^{\prime \prime} \cos v, f^{\prime \prime} \sin v\right), \\
x_{u v} & =\left(0,-f^{\prime} \sin v, f^{\prime} \cos v\right), \\
x_{v v} & =(0,-f \cos v,-f \sin v),
\end{aligned}
$$

we obtain the components of the first and the second fundamental forms of the surface $M$ as follows:

$$
\begin{gathered}
g_{11}=\left\langle x_{u}, x_{u}\right\rangle=1, \\
g_{12}=g_{21}=\left\langle x_{u}, x_{v}\right\rangle=0, \\
g_{22}=\left\langle x_{v}, x_{v}\right\rangle=f^{2}, \\
h_{11}=\left\langle x_{u u}, G\right\rangle=f^{\prime} g^{\prime \prime}-f^{\prime \prime} g^{\prime}=t^{\prime}, \\
h_{12}=h_{21}=\left\langle x_{u v}, G\right\rangle=0, \\
h_{22}=\left\langle x_{v v}, G\right\rangle=f g^{\prime}=f \sinh t,
\end{gathered}
$$


where Gauss map $G$ is defined by $\left(x_{u} \times x_{v}\right) /\left|x_{u} \times x_{v}\right|=$ $\left(-f^{\prime},-g^{\prime} \cos v,-g^{\prime} \sin v\right)$.

So the matrix $\left(h_{i j}\right)$ composed by the second fundamental form $h$ can be expressed as

$$
\left(\begin{array}{ll}
h_{11} & h_{12} \\
h_{21} & h_{22}
\end{array}\right)=\left(\begin{array}{cc}
t^{\prime} & 0 \\
0 & f \sinh t
\end{array}\right)
$$

Since the surface has no parabolic points, so $\mathscr{H}=h_{11} h_{22}-$ $h_{12}^{2}=t^{\prime} f \sinh t \neq 0$ for every $t$. Then the mean curvature $H$ on $M$ is given by

$$
H=\frac{\left(f^{\prime} g^{\prime \prime}-f^{\prime \prime} g^{\prime}\right) f^{2}+f g^{\prime}}{2 f^{2}}=\frac{1}{2}\left(t^{\prime}+\frac{\sinh t}{f}\right) .
$$

By a straightforward computation, the Laplacian $\Delta^{h}$ of the second fundamental form $h$ on $M$ with the help of (4), (35), and (37) turns out to be

$$
\begin{aligned}
\Delta^{h}= & -\frac{1}{t^{\prime}} \frac{\partial^{2}}{\partial u^{2}}-\frac{1}{f \sinh t} \frac{\partial^{2}}{\partial v^{2}} \\
& +\left(\frac{t^{\prime \prime}}{2 t^{\prime 2}}-\frac{\cosh t}{2 f t^{\prime}}-\frac{\cosh t}{2 \sinh t}\right) \frac{\partial}{\partial u} .
\end{aligned}
$$

Accordingly, we get

$$
\Delta^{h} G=\left(\begin{array}{c}
\frac{3}{2} t^{\prime} \cosh t+\left(\frac{\cosh t}{2 f}+\frac{t^{\prime \prime}}{2 t^{\prime}}\right) \sinh t \\
\left(t^{\prime} \sinh t+\frac{t^{\prime \prime}}{2 t^{\prime}} \cosh t-\frac{1}{2 f}+\frac{\sinh ^{2} t}{2 f}+t^{\prime} \frac{\cosh ^{2} t}{2 \sinh t}\right) \cos v \\
\left(t^{\prime} \sinh t+\frac{t^{\prime \prime}}{2 t^{\prime}} \cosh t-\frac{1}{2 f}+\frac{\sinh ^{2} t}{2 f}+t^{\prime} \frac{\cosh ^{2} t}{2 \sinh t}\right) \sin v
\end{array}\right) .
$$

By the assumption (33) and the above equation, we get the following system of differential equations:

$$
\begin{aligned}
& \frac{3}{2} t^{\prime} \cosh t+\left(\frac{\cosh t}{2 f}+\frac{t^{\prime \prime}}{2 t^{\prime}}\right) \sinh t+a_{11} \cosh t \\
& +a_{12} \sinh t \cos v+a_{13} \sinh t \sin v=0, \\
& \left(t^{\prime} \sinh t+\frac{t^{\prime \prime}}{2 t^{\prime}} \cosh t-\frac{1}{2 f}+\frac{\sinh ^{2} t}{2 f}+t^{\prime} \frac{\cosh ^{2} t}{2 \sinh t}\right. \\
& \left.+a_{22} \sinh t\right) \cos v+a_{21} \cosh t+a_{23} \sinh t \sin v=0 \\
& \left(t^{\prime} \sinh t+\frac{t^{\prime \prime}}{2 t^{\prime}} \cosh t-\frac{1}{2 f}+\frac{\sinh ^{2} t}{2 f}+t^{\prime} \frac{\cosh t}{2 \sinh t}\right. \\
& \left.+a_{33} \sinh t\right) \sin v+a_{31} \cosh t+a_{32} \sinh t \cos v=0,
\end{aligned}
$$

where $a_{i j}(i, j=1,2,3)$ denote the components of the matrix $\Lambda$ given by (33). In order to prove the theorem, we have to solve the above system of ordinary differential equations. From (42) we easily deduce that $a_{12}=a_{21}=a_{13}=a_{23}=a_{31}=$ $a_{32}=0$ and $a_{22}=a_{33}$; that is, the matrix $\Lambda$ is diagonal. We put $a_{22}=a_{33}=\lambda$ and $a_{11}=\mu$. Then, the system (42) is reduced to the following equations:

$$
\frac{3}{2} t^{\prime} \cosh t+\left(\frac{\cosh t}{2 f}+\frac{t^{\prime \prime}}{2 t^{\prime}}\right) \sinh t=-\mu \cosh t
$$

$t^{\prime} \sinh t+\frac{t^{\prime \prime}}{2 t^{\prime}} \cosh t-\frac{1}{2 f}+\frac{\sinh ^{2} t}{2 f}+t^{\prime} \frac{\cosh ^{2} t}{2 \sinh t}=-\lambda \sinh t$
By the computation $(43) \times \cosh t-(44) \times \sinh t$, we easily get

$$
\frac{\sinh t}{f}+t^{\prime}=-\mu \cosh ^{2} t+\lambda \sinh ^{2} t .
$$

We discuss five cases according to the constants $\lambda$ and $\mu$.

Case $1(\lambda=\mu=0)$. In this case, we easily get $t^{\prime}+$ $(\sinh t / f)=0$, which implies that the mean curvature $H$ vanishes identically because of (39). Therefore, the surface is minimal; from theorem 1 in [9], it is the 1st kind of catenoid. Furthermore, the 1st kind of catenoid satisfies condition (33).

Case $2(\lambda=\mu \neq 0)$. By (45), we get

$$
t^{\prime}=-\frac{\sinh t}{f}-\lambda
$$

Differentiating (46) with respect to $u$, we have

$$
t^{\prime \prime}=-\frac{t^{\prime} \cosh t}{f}+\frac{\sinh t \cosh t}{f^{2}} .
$$

Combining (46), (47), and (43), we get

$$
\lambda^{2} f^{2}+4 \lambda f \sinh t+4 \sinh ^{2} t=0,
$$

from which

$$
f=-\frac{2 \sinh t}{\lambda} .
$$

Furthermore, (49) together with (46) becomes $t^{\prime}=-\lambda / 2$; that is,

$$
t(u)=-\frac{\lambda}{2} u+k, \quad k \in \mathbb{R} .
$$


On the other hand, by (35) and (50), we get

$$
\begin{gathered}
f(u)=-\frac{2}{\lambda} \sinh \left(-\frac{\lambda}{2} u+k\right), \\
g(u)=-\frac{2}{\lambda} \cosh \left(-\frac{\lambda}{2} u+k\right)+c, \quad c \in \mathbb{R} .
\end{gathered}
$$

Then, the surface $M$ has the following expression:

$$
\begin{aligned}
x(u, v)=( & -\frac{2}{\lambda} \cosh \left(-\frac{\lambda}{2} u+k\right)+c, \\
& {\left[-\frac{2}{\lambda} \sinh \left(-\frac{\lambda}{2} u+k\right)\right] \cos v, } \\
& {\left.\left[-\frac{2}{\lambda} \sinh \left(-\frac{\lambda}{2} u+k\right)\right] \sin v\right), \quad c, k \in \mathbb{R} . }
\end{aligned}
$$

Consequently, we have

$$
\langle x(u, v)-\mathbf{C}, x(u, v)-\mathbf{C}\rangle=-\left(\frac{2}{\lambda}\right)^{2}, \quad \mathbf{C}=(c, 0,0),
$$

which means that the surface $M$ is contained in the hyperbolic pseudosphere $H^{2}(-2 /|\lambda|)$ centered at $\mathbf{C}$ with radius $2 /|\lambda|$. Also, the hyperbolic pseudosphere satisfies condition (33).

Case $3(\lambda \neq 0, \mu=0)$. In this case, $(45)$ becomes $(\sinh t / f)+$ $t^{\prime}=\lambda \sinh ^{2} t$; that is,

$$
t^{\prime}=-\frac{\sinh t}{f}+\lambda \sinh ^{2} t
$$

and thus

$$
t^{\prime \prime}=-\frac{t^{\prime} \cosh t}{f}+\frac{\sinh t \cosh t}{f^{2}}+2 \lambda t^{\prime} \sinh t \cosh t .
$$

Substituting (54) and (55) into (43), we get

$$
5 \lambda^{2} f^{2} \sinh ^{2} t-8 \lambda f \sinh t+4=0 .
$$

Differentiating the above equation, we have

$$
5 \lambda f \sinh t-4=0 \text {. }
$$

If we take the differentiation of the equation once again, we get

$$
\lambda^{2} f \sinh ^{2} t \cosh t=0
$$

Since $f$ is a positive function and $\lambda \neq 0, \cosh t \sinh ^{2} t=0$ for every $t$. Therefore, $t=t(u)$ is vanishing identically for every $u$. Hence, we have

$$
\begin{gathered}
f(u)=u+c, \\
g(u)=a, \quad a, c \in \mathbb{R} .
\end{gathered}
$$

It implies that $M$ is a part of a Euclidean plane $\mathbb{R}^{2}$ whose points are parabolic. Thus, there is no surface of revolution with timelike axis satisfying this case.

Case $4(\lambda=0, \mu \neq 0)$. In this case, (45) becomes $(\sinh t / f)+$ $t^{\prime}=-\mu \cosh ^{2} t$; that is,

$$
t^{\prime}=-\frac{\sinh t}{f}-\mu \cosh ^{2} t
$$

and thus

$$
t^{\prime \prime}=-\frac{t^{\prime} \cosh t}{f}+\frac{\sinh t \cosh t}{f^{2}}-2 \mu t^{\prime} \sinh t \cosh t .
$$

Furthermore, by (43), (60), and (61), we get

$$
\alpha_{1} f^{2}+\alpha_{2} f+\alpha_{3}=0
$$

where we put

$$
\begin{gathered}
\alpha_{1}=\mu^{2}\left(5 \sinh ^{4} t+6 \sinh ^{2} t+1\right), \\
\alpha_{2}=\mu\left(8 \sinh ^{3} t+4 \sinh t\right), \\
\alpha_{3}=4 \sinh ^{2} t .
\end{gathered}
$$

Differentiating (62) and using (60), we find

$$
\beta_{1} f^{2}+\beta_{2} f+\beta_{3}=0,
$$

where

$$
\begin{gathered}
\beta_{1}=\mu^{2}\left(90 \sinh ^{8} t+172 \sinh ^{6} t+104 \sinh ^{4} t+20 \sinh ^{2} t-2\right), \\
\beta_{2}=\mu\left(120 \sinh ^{7} t+120 \sinh ^{5} t+24 \sinh ^{3} t-8 \sinh t\right), \\
\beta_{3}=40 \sinh ^{6} t-8 \sinh ^{2} t .
\end{gathered}
$$

Combining (62) and (64), we can show

$$
\gamma_{1} f+\gamma_{2}=0
$$

where $\gamma_{1}=\alpha_{2} \beta_{1}-\alpha_{1} \beta_{2}, \gamma_{2}=\alpha_{3} \beta_{1}-\alpha_{1} \beta_{3}$.

Differentiating once again this equation and using the same algebraic techniques above, we find the following trigonometric polynomial in $\sinh t$ satisfying

$$
\mu^{2}\left(\sum_{i=1}^{12} c_{i} \sinh ^{8+2 i} t\right)=0,
$$

where $c_{1}=540672, c_{2}=6397952, \ldots, c_{12}=3072000$ are coefficients as nonzero constant of the function $\sinh ^{8+2 i} t$. Since this polynomial is equal to zero for every $t$, all its coefficients must be zero. Thus, we have $\mu=0$. So we get a contradiction, and therefore, in this case, there are no surfaces of revolution with timelike axis.

Case 5 (let $\lambda \neq 0, \mu \neq 0$, and $\lambda \neq \mu$ ). In this case, (45) is unchanged; that is,

$$
t^{\prime}=-\frac{\sinh t}{f}-\mu \cosh ^{2} t+\lambda \sinh ^{2} t
$$


and thus

$$
\begin{aligned}
t^{\prime \prime}= & -\frac{t^{\prime} \cosh t}{f}+\frac{\sinh t \cosh t}{f^{2}}-2 \mu t^{\prime} \sinh t \cosh t \\
& +2 \lambda t^{\prime} \sinh t \cosh t
\end{aligned}
$$

From which, (43) is written as

$$
\xi_{1} f^{2}+\xi_{2} f+\xi_{3}=0
$$

where

$$
\begin{gathered}
\xi_{1}=5 \theta^{2} \sinh ^{4} t-6 \mu \theta \sinh ^{2} t+\mu^{2}, \\
\xi_{2}=-8 \theta \sinh ^{3} t+4 \mu \sinh t, \\
\xi_{3}=4 \sinh ^{2} t, \quad \theta=\lambda-\mu .
\end{gathered}
$$

Differentiating (70) and using (68), we find

$$
\eta_{1} f^{2}+\eta_{2} f+\eta_{3}=0
$$

where

$$
\begin{gathered}
\eta_{1}=90 \theta^{4} \sinh ^{8} t+172 \mu \theta^{3} \sinh ^{6} t+104 \mu^{2} \theta^{2} \sinh ^{4} t \\
+20 \mu^{3} \theta \sinh ^{2} t-2 \mu^{4}, \\
\eta_{2}=-120 \theta^{3} \sinh ^{7} t+120 \mu \theta^{2} \sinh ^{5} t-24 \mu^{2} \theta \sinh ^{3} t \\
-8 \mu^{3} \sinh t, \\
\eta_{3}=40 \theta^{2} \sinh ^{6} t-8 \mu^{2} \sinh ^{2} t .
\end{gathered}
$$

Combining (70) and (72), we have

$$
\zeta_{1} f+\zeta_{2}=0
$$

where $\zeta_{1}=\xi_{2} \eta_{1}-\xi_{1} \eta_{2}, \zeta_{2}=\xi_{3} \eta_{1}-\xi_{1} \eta_{3}$.

Hence, by this procedure, (74) is reduced to a linear one with respect to the function $f$. Therefore, if we repeat this method one more time, we can find the following polynomial:

$$
\sum_{i=1}^{12} c_{i} \theta^{2+i} \mu^{12-i} \sinh ^{8+2 i} t
$$

where $c_{1}=258048, c_{2}=5046272, \ldots, c_{12}=3072000$ are nonzero constants. Since this polynomial is equal to zero for every $t$, all its coefficients must be zero. Therefore we conclude that $\theta=0$; that is, $\lambda=\mu$, which is a contradiction. Consequently, there are no surfaces of revolution with timelike axis in this case.

When $\varepsilon=-1, M$ is a timelike surface. In this case, we can assume that $f^{\prime}(u)=\sinh t$ and $g^{\prime}(u)=\cosh t$, and using the same algebraic techniques as for $\varepsilon=1$ easily prove that the 3rd kind of catenoid and the de Sitter pseudosphere satisfy condition (33). This completes the proof.

\section{The Surface of Revolution with Spacelike Axis}

In this section, we will classify the surfaces of revolution with spacelike axis in $\mathbb{R}_{1}^{3}$ that satisfy condition (3).

Theorem 9. The only surfaces of revolution with spacelike axis in $\mathbb{R}_{1}^{3}$ whose Gauss map $G$ satisfies

$$
\Delta^{h} G=\Lambda G, \quad \Lambda \in \operatorname{Mat}(3, \mathbb{R})
$$

are locally the 2nd kind of catenoid, the 4th kind of catenoid, the 5th kind of catenoid, the hyperbolic pseudosphere, or the de Sitter pseudosphere.

Proof. Let $M$ be a surface of revolution with spacelike axis as (9). We may assume that the profile curve $\gamma$ is of unit speed; thus

$$
\left\langle\gamma^{\prime}, \gamma^{\prime}\right\rangle=f^{\prime 2}(u)+g^{\prime 2}(u)=1
$$

Then we may put

$$
f^{\prime}(u)=\cos t, \quad g^{\prime}(u)=\sin t
$$

for the smooth function $t=t(u)$. Using the natural frame $\left\{x_{u}, x_{v}\right\}$ of $M$ defined by

$$
\begin{gathered}
x_{u}=\left(f^{\prime} \sinh v, f^{\prime} \cosh v, g^{\prime}\right), \\
x_{v}=(f \cosh v, f \sinh v, 0), \\
x_{u u}=\left(f^{\prime \prime} \sinh v, f^{\prime \prime} \cosh v, g^{\prime \prime}\right), \\
x_{u v}=\left(f^{\prime} \cosh v, f^{\prime} \sinh v, 0\right), \\
x_{v v}=(f \sinh v, f \cosh v, 0),
\end{gathered}
$$

we obtain the components of the first and the second fundamental forms of the surface as follows:

$$
\begin{gathered}
g_{11}=\left\langle x_{u}, x_{u}\right\rangle=1, \quad g_{12}=g_{21}=\left\langle x_{u}, x_{v}\right\rangle=0, \\
g_{22}=\left\langle x_{v}, x_{v}\right\rangle=-f^{2}, \\
h_{11}=\left\langle x_{u u}, G\right\rangle=f^{\prime \prime} g^{\prime}-f^{\prime} g^{\prime \prime}=-t^{\prime}, \\
h_{12}=h_{21}=\left\langle x_{u v}, G\right\rangle=0, \\
h_{22}=\left\langle x_{v v}, G\right\rangle=f g^{\prime}=f \sin t,
\end{gathered}
$$

where Gauss map $G$ is $\left(x_{u} \times x_{v}\right) /\left|x_{u} \times x_{v}\right|=$ $\left(g^{\prime} \sinh v, g^{\prime} \cosh v,-f^{\prime}\right)$. 
So the matrix $\left(h_{i j}\right)$ composed by the second fundamental form $h$ can be expressed as

$$
\left(\begin{array}{ll}
h_{11} & h_{12} \\
h_{21} & h_{22}
\end{array}\right)=\left(\begin{array}{cc}
-t^{\prime} & 0 \\
0 & f \sin t
\end{array}\right)
$$

Since the surface has no parabolic points, so $\mathscr{H}=h_{11} h_{22}-$ $h_{12}^{2}=t^{\prime} f \sin t \neq 0$ for every $t$. Then the mean curvature $H$ on $M$ is given by

$$
H=\frac{\left(f^{\prime \prime} g^{\prime}-f^{\prime} g^{\prime \prime}\right)\left(-f^{2}\right)+f g^{\prime}}{2\left(-f^{2}\right)}=-\frac{1}{2}\left(t^{\prime}+\frac{\sin t}{f}\right) \text {. }
$$

By a straightforward computation, the Laplacian $\Delta^{h}$ of the second fundamental form $h$ on $M$ with the help of (4), (78), and (80) turns out to be

$$
\begin{aligned}
\Delta^{h}= & \frac{1}{t^{\prime}} \frac{\partial^{2}}{\partial u^{2}}-\frac{1}{f \sin t} \frac{\partial^{2}}{\partial v^{2}} \\
& +\left(-\frac{t^{\prime \prime}}{2 t^{\prime 2}}+\frac{\cos t}{2 f t^{\prime}}+\frac{\cos t}{2 \sin t}\right) \frac{\partial}{\partial u} .
\end{aligned}
$$

Accordingly, we get

$$
\Delta^{h} G=\left(\begin{array}{c}
\left(-t^{\prime} \sin t+\frac{t^{\prime \prime}}{2 t^{\prime}} \cos t-\frac{1}{2 f}-\frac{\sin ^{2} t}{2 f}+t^{\prime} \frac{\cos ^{2} t}{2 \sin t}\right) \sinh v \\
\left(-t^{\prime} \sin t+\frac{t^{\prime \prime}}{2 t^{\prime}} \cos t-\frac{1}{2 f}-\frac{\sin ^{2} t}{2 f}+t^{\prime} \frac{\cos ^{2} t}{2 \sin t}\right) \cosh v \\
\frac{3}{2} t^{\prime} \cos t+\left(\frac{\cos t}{2 f}+\frac{t^{\prime \prime}}{2 t^{\prime}}\right) \sin t
\end{array}\right) .
$$

By the assumption (76) and the above equation, we get the following system of differential equations:

$$
\begin{gathered}
\left(-t^{\prime} \sin t+\frac{t^{\prime \prime}}{2 t^{\prime}} \cos t-\frac{1}{2 f}-\frac{\sin ^{2} t}{2 f}+t^{\prime} \frac{\cos ^{2} t}{2 \sin t}\right. \\
\left.-a_{11} \sin t\right) \sinh v-a_{12} \sin t \cosh v+a_{13} \cos t=0, \\
\left(-t^{\prime} \sin t+\frac{t^{\prime \prime}}{2 t^{\prime}} \cos t-\frac{1}{2 f}-\frac{\sin ^{2} t}{2 f}+t^{\prime} \frac{\cos ^{2} t}{2 \sin t}\right. \\
\left.-a_{22} \sin t\right) \cosh v-a_{21} \sin t \sinh v+a_{23} \cos t=0, \\
\frac{3}{2} t^{\prime} \cos t+\left(\frac{\cos t}{2 f}+\frac{t^{\prime \prime}}{2 t^{\prime}}\right) \sin t-a_{31} \sin t \sinh v \\
-a_{32} \sin t \cosh v+a_{33} \cos t=0,
\end{gathered}
$$

where $a_{i j}(i, j=1,2,3)$ denote the components of the matrix $\Lambda$ given by (76). In order to prove the theorem, we have to solve the above system of ordinary differential equations. From (85) we easily deduce that $a_{12}=a_{21}=a_{13}=a_{23}=a_{31}=$ $a_{32}=0$ and $a_{11}=a_{22}$; that is, the matrix $\Lambda$ is diagonal. We put $a_{11}=a_{22}=\lambda$ and $a_{33}=\mu$. Then, the system (85) is reduced to the following equations:

$$
\begin{gathered}
\frac{3}{2} t^{\prime} \cos t+\left(\frac{\cos t}{2 f}+\frac{t^{\prime \prime}}{2 t^{\prime}}\right) \sin t=-\mu \cos t, \\
-t^{\prime} \sin t+\frac{t^{\prime \prime}}{2 t^{\prime}} \cos t-\frac{1}{2 f}-\frac{\sin ^{2} t}{2 f}+t^{\prime} \frac{\cos ^{2} t}{2 \sin t}=\lambda \sin t .
\end{gathered}
$$

By the computation $(87) \times \sin t-(86) \times \cos t$, we easily get

$$
-\frac{\sin t}{f}-t^{\prime}=\lambda \sin ^{2} t+\mu \cos ^{2} t
$$

We discuss five cases according to the constants $\lambda$ and $\mu$.
Case $1(\lambda=\mu=0)$. In this case, we easily get $-t^{\prime}-$ $(\sin t / f)=0$, which implies that the mean curvature $H$ vanishes identically because of (82). Therefore, the surface is minimal; from theorem 2 in [9], it is the 5th kind catenoid. Furthermore, the 5 th kind catenoid satisfies the condition (76).

Case $2(\lambda=\mu \neq 0)$. By (88), we get

$$
t^{\prime}=-\frac{\sin t}{f}-\lambda
$$

Differentiating (89) with respect to $u$, we have

$$
t^{\prime \prime}=-\frac{t^{\prime} \cos t}{f}+\frac{\sin t \cos t}{f^{2}} .
$$

Combining (89), (90), and (86), we get

$$
\lambda^{2} f^{2}+4 \lambda f \sin t+4 \sin ^{2} t=0
$$

from which

$$
f=-\frac{2 \sin t}{\lambda}
$$

Furthermore, (92) together with (89) becomes $t^{\prime}=-\lambda / 2$; that is,

$$
t(u)=-\frac{\lambda}{2} u+k, \quad k \in \mathbb{R} .
$$

On the other hand, by (78) and (93), we have

$$
\begin{gathered}
f(u)=-\frac{2}{\lambda} \sin \left(-\frac{\lambda}{2} u+k\right), \\
g(u)=\frac{2}{\lambda} \cos \left(-\frac{\lambda}{2} u+k\right)+c, \quad c \in \mathbb{R} .
\end{gathered}
$$


Then, the surface $M$ has the following expression:

$$
\begin{aligned}
x(u, v)=( & {\left[-\frac{2}{\lambda} \sin \left(-\frac{\lambda}{2} u+k\right)\right] \sinh v, } \\
& {\left[-\frac{2}{\lambda} \sin \left(-\frac{\lambda}{2} u+k\right)\right] \cosh v, } \\
& \left.\frac{2}{\lambda} \cos \left(-\frac{\lambda}{2} u+k\right)+c\right), \quad c, k \in \mathbb{R} .
\end{aligned}
$$

Consequently, we have

$$
\langle x(u, v)-\mathbf{C}, x(u, v)-\mathbf{C}\rangle=\left(\frac{2}{\lambda}\right)^{2}, \quad \mathbf{C}=(0,0, c),
$$

which means that the surface $M$ is contained in the de Sitter pseudosphere $S_{1}^{2}(2 /|\lambda|)$ centered at $\mathbf{C}$ with radius $2 /|\lambda|$. Also, the de Sitter pseudosphere satisfies condition (76). In the cases of 3, 4, and 5, we will use the same method of Section 3 and easily get that there are no surfaces of revolution with spacelike axis satisfies condition (76).

When the surface of revolution has the expression given by (10), we can similarly prove that the 2 nd kind of catenoid, 4th kind of catenoid, the de Sitter pseudosphere, and the hyperbolic pseudosphere satisfy condition (76). This completes the proof.

\section{Conflict of Interests}

The authors declare that there is no conflict of interests in this work.

\section{Acknowledgments}

The authors were supported by NSF of China (no. 11271063) and Youth Science Foundation of Heilongjiang Institute of Technology (no. 2012QJ19).

\section{References}

[1] B. Y. Chen, Total Mean Curvature and Submanifolds of Finite Type, World Scientific, Singapore, 1984.

[2] B. Y. Chen and P. Piccinni, "Submanifolds with finite type Gauss map," Bulletin of the Australian Mathematical Society, vol. 35, no. 2, pp. 161-186, 1987.

[3] E. A. Ruh and J. Vilms, "The tension field of the Gauss map," Transactions of the American Mathematical Society, vol. 149, pp. 569-573, 1970.

[4] C. Jang, "Surfaces with 1-type Gauss map," Kodai Mathematical Journal, vol. 19, pp. 388-394, 1996.

[5] F. Dillen, J. Pas, and L. Verstralen, "On the Gauss map of surfaces of revolution," Bulletin of the Institute of Mathematics Academia Sinica, vol. 18, pp. 239-249, 1990.

[6] S. M. Choi, "On the Gauss map of surfaces of revolution in a 3-dimensional Minkowski space," Tsukuba Journal of Mathematics, vol. 19, pp. 351-367, 1995.

[7] M. Jin, D. Pei, and S. Xu, "On the gauss map of survaces of revolution with lightlike axis in Minkowski 3-space," Journal of Function Spaces and Applications, vol. 2013, Article ID 130495, 8 pages, 2013.
[8] C. C. Beneki, G. Kaimakamis, and B. J. Papantoniou, "Helicoidal surfaces in three-dimensional Minkowski space," Journal of Mathematical Analysis and Applications, vol. 275, no. 2, pp. 586614, 2002.

[9] I. V. de Woestijne, "Minimal surfaces in the 3-dimensional Minkowski space," in Geometry and Topology of Submanifolds:II, pp. 344-369, World Scientific, Singapore, 1990. 


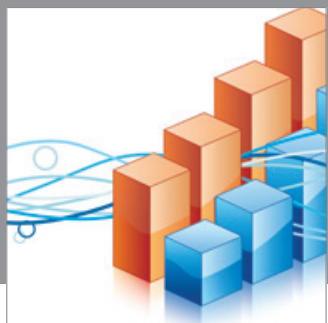

Advances in

Operations Research

mansans

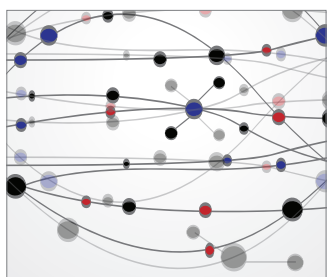

The Scientific World Journal
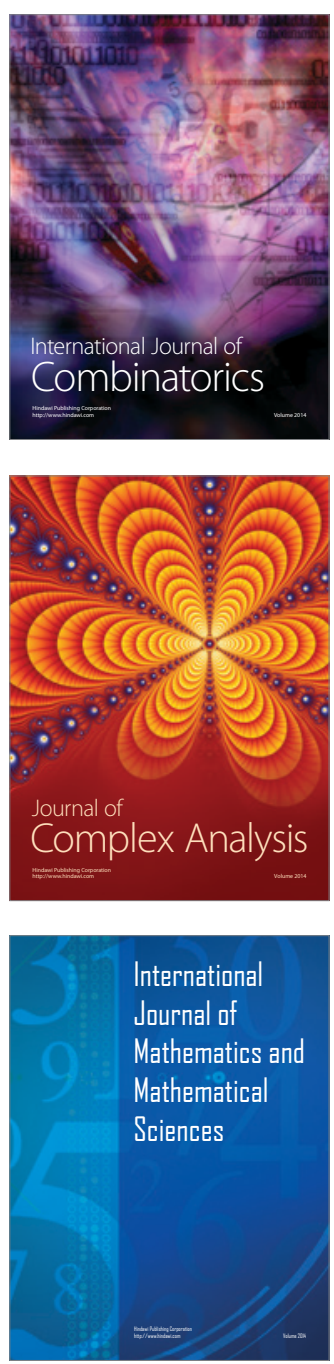
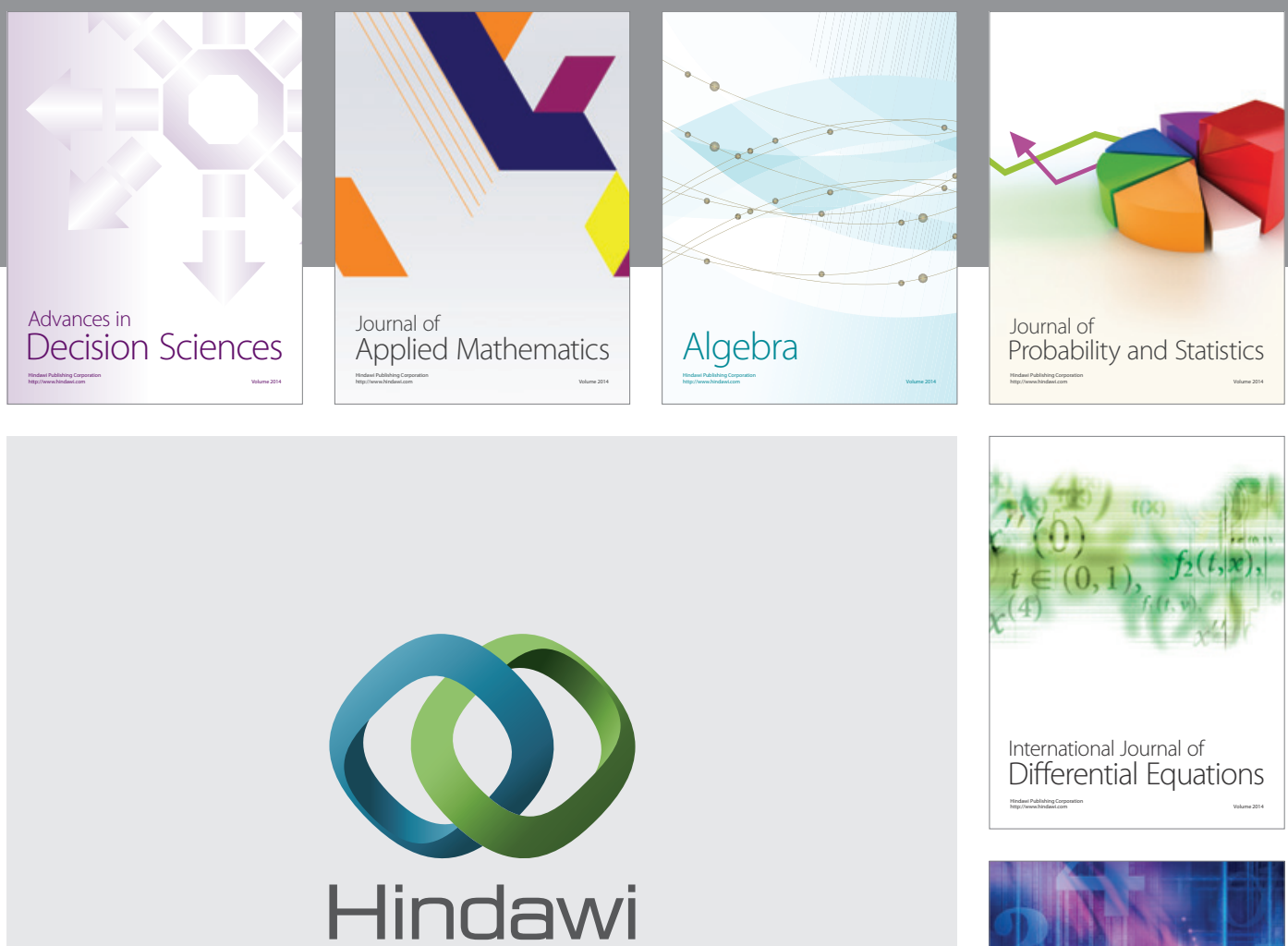

Submit your manuscripts at http://www.hindawi.com
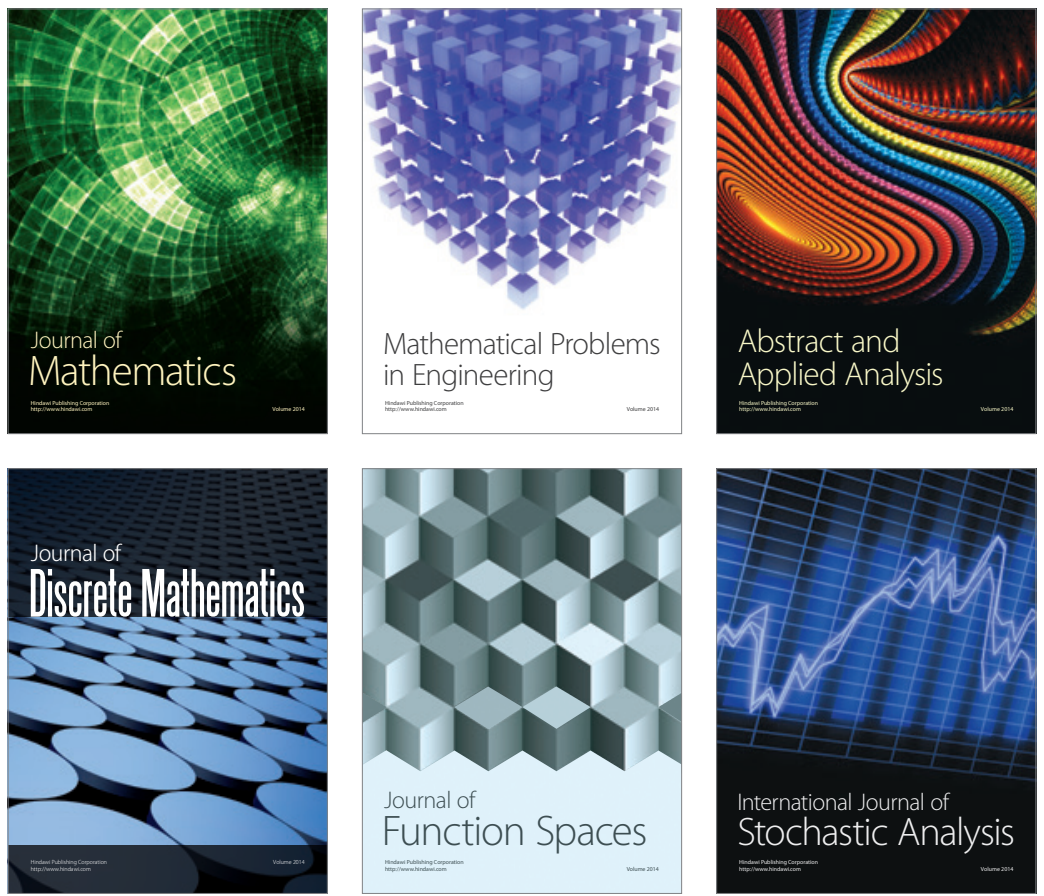

Journal of

Function Spaces

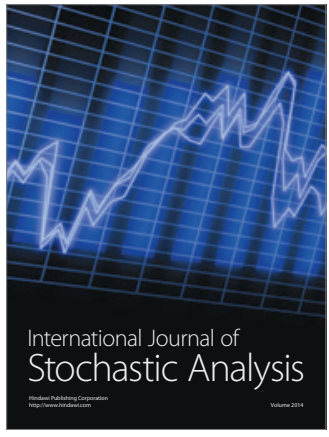

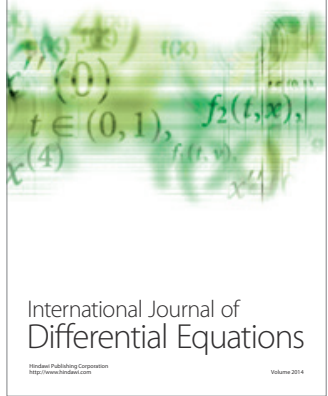
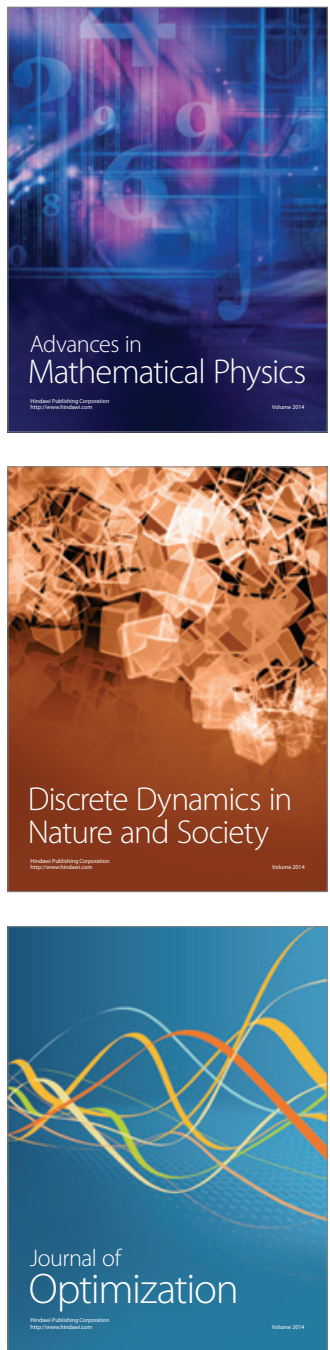